\title{
Risk of Contrast-Induced Nephropathy after Repeated Contrast Medium Administration
}

\author{
Violeta Dinesch ${ }^{\star \star}$, Mihail Dinesch², Cosmin Macarie ${ }^{3}$, lleana Voichita Sirbu, Mircea Buruian4 \\ 1. University of Medicine and Pharmacy Tirgu Mures, Romania \\ 2. Department of Cardiology, Institute for Cardiovascular Disease and Heart Transplant, Tirgu Mures, Romania \\ 3. Department of Internal Medicine M3, University of Medicine and Pharmacy, Department of Cardiology, Institute for Cardiovascular Disease and Heart \\ Transplant, Tirgu Mures, Romania \\ 4. Department of Morphologic Sciences M1, University of Medicine and Pharmacy, Tirgu Mures, Romania
}

\begin{abstract}
Objective: Non-invasive coronary computed tomography angiography is frequently used to exclude coronary artery disease in patients with low-to-intermediate pre-test probability because of the high negative predictive value. The strategy of coronary computed tomography angiography and subsequent invasive coronary angiography in case of positive findings has risks owing to repeated contrast medium administration and the possibility of contrast-induced nephropathy. Methods: We retrospectively evaluated the changes in the serum creatinine level and estimated glomerular filtration rate (at baseline, $24 \mathrm{~h}$, and $48 \mathrm{~h}$ after contrast administration) in patients with repeated contrast medium administration in order to evaluate contrast-induced nephropathy development. All patients were intravenously hydrated with $1000 \mathrm{ml}$ sodium chloride (0.9\%) per day during hospitalization. Results: The study included 17 patients. Of these patients, 7 (41.2\%) had prior impaired renal function (estimated glomerular filtration rate $<60 \mathrm{ml} / \mathrm{min} / 1.73 \mathrm{~m} 2$ ). The mean coronary computed tomography angiography contrast medium (iopromide $769 \mathrm{mg} / \mathrm{ml}$ ) volume was $114.11 \pm 7.75 \mathrm{ml}$ and the mean invasive coronary angiography contrast medium (iohexol $755 \mathrm{mg} / \mathrm{ml}$ ) volume was $129.7 \pm 19.24 \mathrm{ml}$. The serum creatinine level was significantly higher and the estimated glomerular filtration rate was significantly lower at 48 hours after the second contrast medium administration than at baseline ( $p=0.05$ and $p=0.03$, respectively). None of the patients had contrast-induced nephropathy. Conclusion: Repeated contrast medium administration was not associated with contrast-induced nephropathy development at 48 hours after the second contrast medium administration, even in patients with prior impaired renal function.
\end{abstract}

Keywords: contrast-induced nephropathy, contrast medium administration, renal function

Received 28 July 2018 / Accepted 22 August 2018

\section{Introduction}

The rapid evolution of cardiovascular imaging during the last few decades has resulted in an increase in the use of intra-venous/intra-arterial iodinated contrast agents. Conventional invasive coronary angiography (ICA) is the gold standard approach for the evaluation of coronary artery disease $(\mathrm{CAD})$, and non-invasive coronary computed tomography angiography (CCTA) is frequently used to exclude CAD in patients with low-to-intermediate pre-test probability. However, the strategy of CCTA and subsequent ICA in the case of positive findings has some risks owing to repeated contrast exposure and the possibility of subsequent contrast-mediated renal injury. Contrast-induced nephropathy (CIN) was first described in the 1950s [1], and it remains one of the leading causes of hospitalacquired acute renal injury [2]. Several studies have mentioned the incidence of CIN after single administration of radiocontrast medium $[3,4]$. However, in real life, repeated contrast medium administration (CMA) is not infrequent. The present study aimed to assess the change in renal function after two consecutive imaging procedures involving intra-venous and intra-arterial CMA in order to evaluate CIN development. To our knowledge, the risk of CIN development after CCTA followed by ICA has not been investigated previously.

\footnotetext{
* Correspondence to: Violeta Dinesch
}

E-mail: doc_violeta@yahoo.com

\section{Methods}

We reviewed the records of patients admitted to our institution for CCTA followed by ICA between January and December 2015. All study participants provided informed consent, and the study design was approved by the appropriate ethics review board.

Renal function was evaluated according to changes in the serum creatinine $(\mathrm{sCr})$ level and eGFR 24 hours after each CMA and 48 hours after the last CMA compared with baseline values (before CMA). The diagnostic criterion for $\mathrm{CIN}$ was a rise in the $\mathrm{s} C \mathrm{r}$ level by $25 \%$ or more or an absolute increase in the $\mathrm{s} \mathrm{Cr}$ level by $0.5 \mathrm{mg} / \mathrm{dl}$ or more compared with the baseline value. The eGFR was calculated using the Cockroft-Gault formula (creatinine clearance $[\mathrm{CrCl}]=[140$-age $] \times$ weight $/ \mathrm{s} \mathrm{Cr} \times 72 ; \mathrm{CrCl}_{\text {female }}=\mathrm{CrCl}$ $\times 0.85$ [female sex adjustment]). The results are expressed as mean \pm standard deviation (SD), and the data were compared using one-way ANOVA for repeated measurements. All statistical analyses were performed using STATA 14.0 (Stata Corporation, College Station, TX, USA). A p-value $\leq 0.05$ was considered significant.

\section{Results}

The study included 17 patients. Prior impaired renal function (eGFR $\left.<60 \mathrm{ml} / \mathrm{min} / 1.73 \mathrm{~m}^{2}\right)$ was noted in $41.2 \%$ of the patients, and a history of ST-elevation myocardial infarction (STEMI) was noted in $41.2 \%$ of the patients. 
The demographic and clinical characteristics of the study patients are presented in Table I.

Table I. Demographic and clinical characteristics of the patients

\begin{tabular}{lc}
\hline Parameter & Number (\%) \\
\hline Male & $16(94.1)$ \\
Age (mean \pm SD), years & $61.41 \pm 9.007$ \\
Hypertension & $15(88.2)$ \\
Diabetes mellitus & $4(23.5)$ \\
Hypercholesterolemia & $6(35.2)$ \\
Smoking history & $5(29.4)$ \\
Obesity & $4(23.5)$ \\
Prior STEMI & $7(41.2)$ \\
Prior non-STEMI & $2(11.8)$ \\
eGFR $<60$ ml/min/1.73 m² & $7(41.2)$ \\
\hline eGFR: estimated glomerular filtration rate; STEMI: ST-elevation myocardial infarction
\end{tabular}

The time interval between procedures was 24 hours. In patients with a prior eGFR $<60 \mathrm{ml} / \mathrm{min} / 1.73 \mathrm{~m}^{2}$, ICA was performed after an additional 24-hour period. All patients were intravenously hydrated with $1000 \mathrm{ml}$ sodium chloride $(0.9 \%)$ per day during hospitalisation. Oral fluid intake was not assessed. Iopromide $(769 \mathrm{mg} / \mathrm{ml})$ was used for CCTA, and iohexol $(755 \mathrm{mg} / \mathrm{ml})$ was used for ICA.

The mean contrast volume received was $114.11 \pm 7.75$ $\mathrm{ml}$ for iopromide and $129.7 \pm 19.24 \mathrm{ml}$ for iohexol.

The $s$ Cr levels and eGFRs at baseline, 24 hours after the first CMA (CCTA), and 24 and 48 hours after the second CMA (ICA) are shown in Table II.

There were no significant differences in the mean $\mathrm{sCr}$ level and mean eGFR between baseline and 24 hours after ICA (sCr: $0.92 \pm 0.28$ vs. $0.92 \pm 0.33 \mathrm{mg} / \mathrm{dl}, \mathrm{F}(2.32)=$ 1.6, $\mathrm{p}=0.21$; eGFR: $95.43 \pm 26.69$ vs. $94.48 \pm 23.3 \mathrm{ml} /$ $\left.\mathrm{min} / 1.73 \mathrm{~m}^{2}, \mathrm{~F}(2.32)=1.22, \mathrm{p}=0.29\right)$. The $\mathrm{sCr}$ level was significantly higher and the eGFR was significantly lower 48 hours after ICA than at baseline ( $s$ Cr: $0.95 \pm 0.08$ vs. $0.92 \pm 0.28 \mathrm{mg} / \mathrm{dl}, \mathrm{F}(3.48)=3.08, \mathrm{p}=0.05$; eGFR: 91.82 \pm 21.84 vs. $95.43 \pm 26.69 \mathrm{ml} / \mathrm{min} / 1.73 \mathrm{~m}^{2}, \mathrm{~F}(3.48)=$ $4.13, \mathrm{p}=0.03)$. None of the patients met the diagnostic criterion for CIN.

\section{Discussion}

CIN affects up to $50 \%$ of patients at high risk [3], and it is a clinical reality with high health and economic burdens [5]. In a previous large meta-analysis, James et al. found that the presence of CIN following coronary angiography was associated with increased patient mortality and major cardiovascular events [6].

The risk of CIN after a second contrast exposure has been investigated in a few studies. Trivedi et al. reported a CIN incidence of $14.3 \%$ after repeated CMA, even in patients with preserved renal function [7]. On the other hand, Winther et al. performed a study on the effect of repeated CMA in patients with end-stage kidney disease and found a low risk of post-contrast acute kidney injury and long-term complications [8].

In the present study, we investigated the impact of both intra-venous and intra-arterial CMA on renal function assessed according to the sCr level and eGFR. The important finding of our study was the complete absence of CIN, even in patients with prior impaired renal function. Only 1 patient showed a significant decrease in the eGFR, resulting in a change in the classification of kidney disease from $3 \mathrm{a}$ to $3 \mathrm{~b}$. However, this patient had other risk factors for kidney disease, such as hypertension and insulindependent diabetes mellitus. Interestingly, several patients showed better values of sCr and eGFR at 24 hours after the first CMA, supporting the hypothesis of the correction of pre-renal dysfunction after the initial procedure by intravenous administration of sodium chloride $(0.9 \%)$. It is known that oral hydration can improve renal function after CMA $[9,10]$. However, data on the extent of oral fluid intake before and after CMA were not available.

Our findings appear to confirm previous results indicating the lack of kidney injury after CMA [11,12]. Sinert et al. compared contrast-exposed patients with contrastunexposed patients and did not find significant kidney injury after CMA in patients with previously normal renal function. In fact, the incidence of acute kidney injury was greater among patients without CMA than among those with CMA (8.9\% vs. 5.7\%) [13]. Additionally, McDonald et al. did not find a greater risk of nephropathy development in contrast-exposed patients than in contrastunexposed patients, irrespective of baseline renal function [14]. These findings question whether CMA or other pathological conditions actually cause degradation of renal function.

The present study had several limitations. First, this study had a small sample size. Second, this retrospective study had a possible selection bias (oral hydration status and other prophylactic treatments to prevent CIN). Third, the $\mathrm{sCr}$ level and eGFR at 72 hours or more after the second CMA were not assessed. Renal function might decline late after CMA. Thus, further studies with a large sample size and long assessment period are needed.

In conclusion, although the $\mathrm{sCr}$ level was high and eGFR was low 48 hours after the second CMA, repeated CMA was not associated with CIN development at this point, even in patients with impaired renal function prior to CMA. Intravenous administration of sodium chloride $(0.9 \%)$ might help improve renal function before and after CMA.

Table II. Evolution of serum creatinine (sCr) level and estimated glomerular filtration rate (eGFR)

\begin{tabular}{|c|c|c|c|c|}
\hline Parameter & Baseline & 24 hours after CCTA & 24 hours after ICA & 48 hours after ICA \\
\hline $\mathrm{sCr}($ mean $\pm \mathrm{SD}), \mathrm{mg} / \mathrm{dl}$ & $0.92 \pm 0.28$ & $0.89 \pm 0.25$ & $0.92 \pm 0.33$ & $0.95 \pm 0.08$ \\
\hline eGFR (mean $\pm \mathrm{SD}), \mathrm{ml} / \mathrm{min} / 1.73 \mathrm{~m} 2$ & $95.43 \pm 26.69$ & $97.68 \pm 23.95$ & $94.48 \pm 23.3$ & $91.82 \pm 21.84$ \\
\hline
\end{tabular}




\section{Author's contribution}

VD - Conceptualization, data curation, formal analysis, methodology, supervision, validation, visualization, writing original draft, writing review and editing

MD - Data curation, investigation, methodology, validation, writing review and editing

CM - Conceptualization, formal analysis, methodology, validation, writing original draft

IVS - Data curation, formal analysis, methodology, supervision, writing review and editing

MB - Conceptualization, formal analysis, methodology;, supervision, validation, writing review and editing

\section{Acknowledgements}

This research did not receive any specific grant from funding agencies in the public, commercial, or not-for-profit sectors.

\section{Conflict of interest}

None to declare.

\section{References}

1. Alwall N, Johnsson S, Tornberg A, et al. Acute renal failure following angiography especially the risk of repeated examination, revealed by eight cases (two deaths). Acta Chir Scand. 1955:109:11-19

2. Mohammed NM, Mahfouz A, Achkar K, et al. Contrast-induced nephropathy. Heart Views. 2013;14:106-116

3. Mehran R, Nikolsky E. Contrast-induced nephropathy: definition, epidemiology, and patients at risk. Kidney Int Suppl. 2006;100:S11-15

4. Rihal CS, Textor SC, Grill DE, et al. Incidence and prognostic importance of acute renal failure after percutaneous coronary intervention. Circulation. 2002;105:2259-2264

5. Brillet, G, Aubry, P, Schmidt, A, Catella, et al. Hospital costs of contrastinduced nephropathy. Value Health. 2015;18:A510

6. James MT, Samuel SM, Manning MA, et al. Contrast-induced acute kidney injury and risk of adverse clinical outcomes after coronary angiography: a systematic review and meta-analysis. Circ Cardiovasc Interv. 2013;6:37-43

7. Trivedi $H$, Foley WD. Contrast-induced nephropathy after a second contrast exposure. Ren Fail. 2010;32:796-801

8. Winther S, Svensson M, Jørgensen HS, et al. Repeated contrast administration is associated with low risk of postcontrast acute kidney injury and long-term complications in patients with severe chronic kidney disease. Am J Transplant. 2016;16:897-907

9. Yoshikawa D, Isobe S, Sato K, et al. Importance of oral fluid intake after coronary computed tomography angiography: an observational study. Eur J Radiol. 2011;77:118-122

10. Rear R, Bell RM, Hausenloy DJ. Contrast-induced nephropathy following angiography and cardiac interventions. Heart. 2016;0:1-11.

11. Hinson JS, Ehmann MR, Fine DM et al. Risk of acute kidney injury after intravenous contrast media admnistration. Ann Emerg Med. 2017;69:577-586

12. McDonald JS, McDonald RJ, Comin $J$ et al. Frequency of acute kidney injury following intravenous contrast medium administration: a systematic review and meta-analysis. Radiology. 2013;267:119-128.

13. Sinert R, Brandler E, Subramanian RA, et al. Does the current definition of contrast-induced acute kidney injury reflect a true clinical entity? Acad Emerg Med. 2012;19: 1261-1267

14. McDonald RJ, McDonald JS, Bida JP, et al. Intravenous contrast material-induced nephropathy: causal or coincident phenomenon? Radiology. 2013;267:106-118 